\title{
Safety of topical vitamin K1 for epidermal growth factor receptor inhibitor (EGFRI)-induced rash
}

\author{
Julie Ryan Wolf 1 , Braden Candela ${ }^{2}$, Ramina Nabiee ${ }^{3}$, Shadi Nassairirad ${ }^{3}$, Reza Mehvar ${ }^{3}$, \\ Hamidreza Montezari ${ }^{3}$, Siu Fun Wong ${ }^{3}$ \\ ${ }^{1}$ Dermatology, ${ }^{2}$ School of Medicine \& Dentistry, University of Rochester Medical Center, Rochester, NY, USA, \\ ${ }^{3}$ Chapman School of Pharmacy, Irvine, CA, USA
}

THE HOPE

FOUNDATION

FOR CANCER RESEARCH

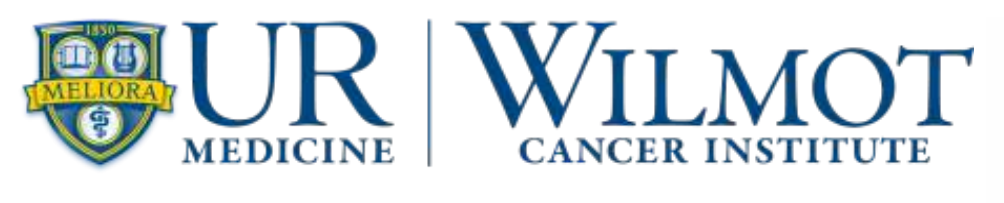

MEDICINE of THE HIGHEST ORDER

\section{INTRODUCTION}

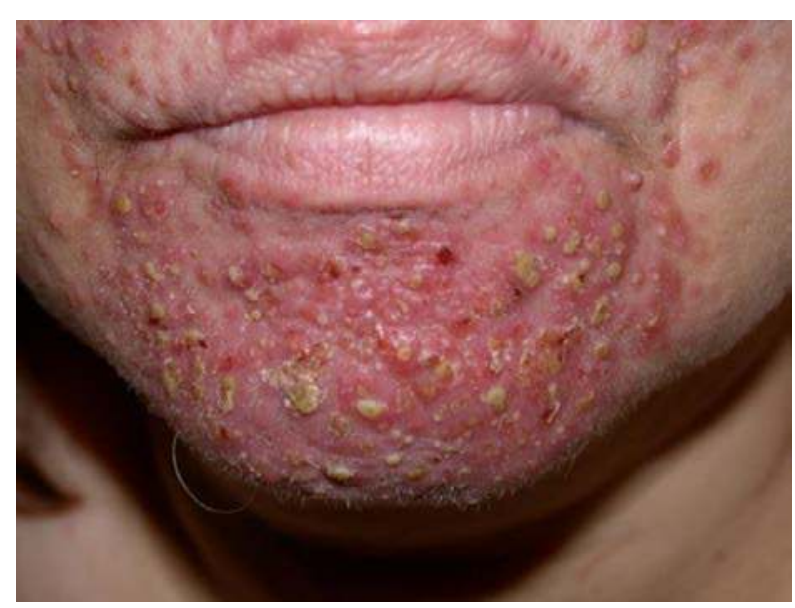

\section{Study Objective:}

Evaluate the safety of a topical $0.1 \%$ vitamin $\mathrm{K} 1$ product for potential use in patients with EGFRI-induced rash.

Palpulopustular rash occurs in $80 \%$ of patients EGFRI for cancer treatment.

Rash often results in decreased quality of life and discontinuation of EGFRI therapy.

Topical Vitamin K1, a phosphatase inhibitor, could reduce rash severity in skin without systemic interference of EFGR inhibition in the tumor.

Topical $0.1 \%$ vitamin $\mathrm{K} 1$ product evaluated had the least systemic absorption potential \& most cosmetically desirable formulation.

\section{METHODS}

\section{Healthy Volunteer Pilot Trial}

10 health volunteers (Caucasian, Female, age range $=22-65$ years) Topical vitamin K1 applied to forearm every 12 hours for five doses without (Phase 1) or with (Phase 2) tape-stripping.

Blood (serum \& plasma) collected at 0,26 , and 50 hours in both Phase $1 \& 2$

> Human Vitamin K1 ELISA (MyBioSource).

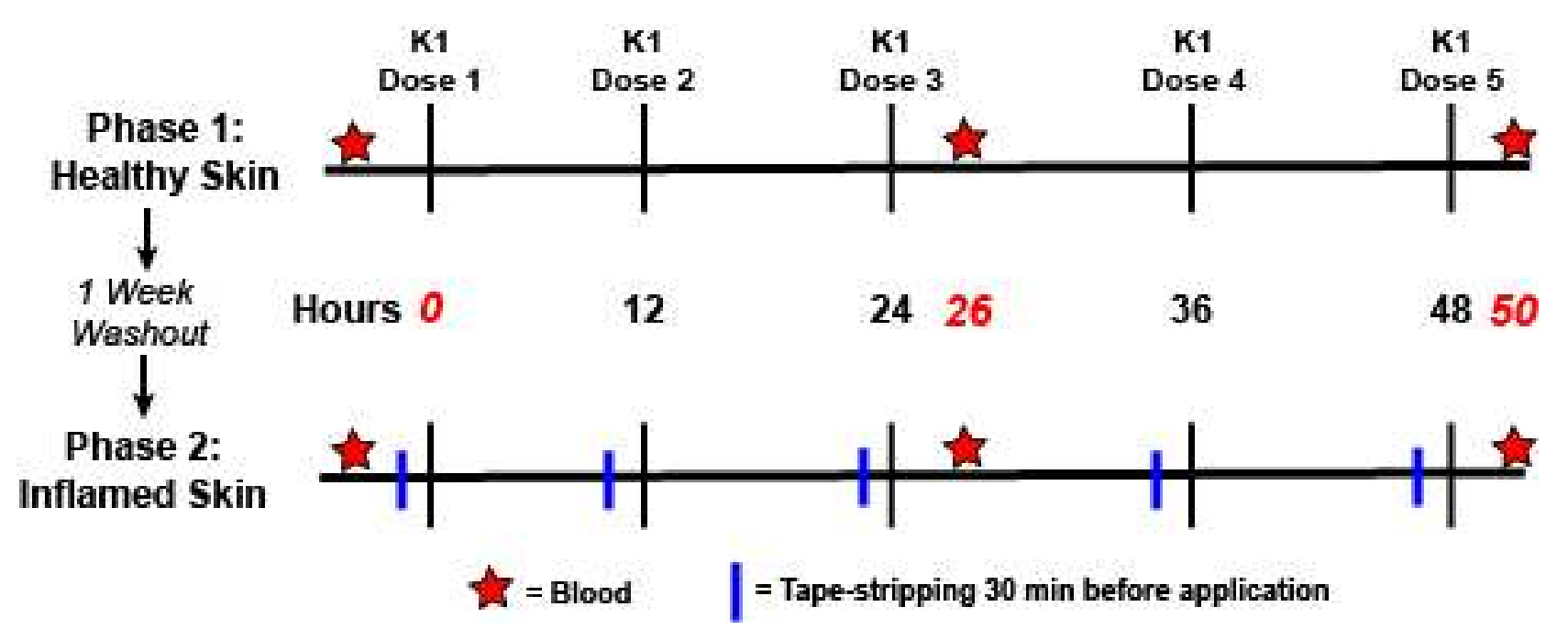

\section{In vitro Study}

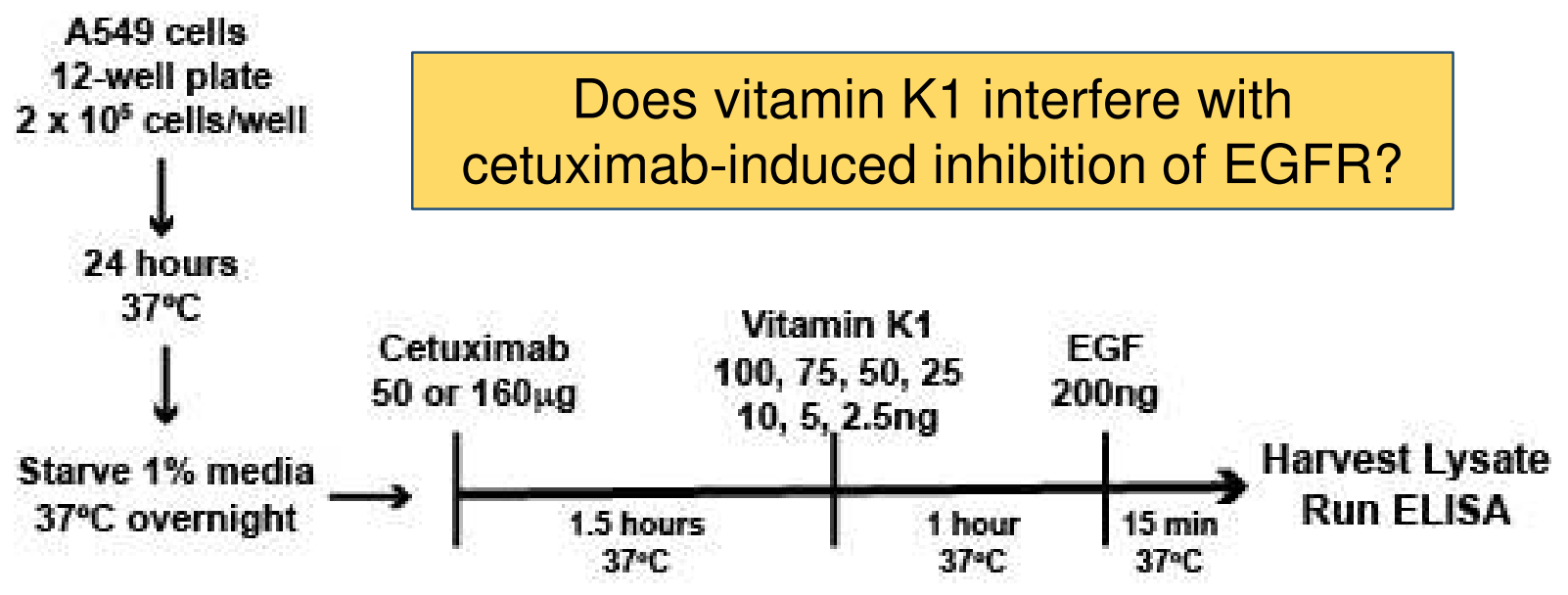

A549 cells cultured at $37^{\circ} \mathrm{C}\left(5 \% \mathrm{CO}_{2}\right)$ in RPMI media $(+10 \%$ FBS (heat-inactivated) +penicillin/streptomycin/glutamine).

Semi-quantitative Human Phospho-EGFR (Tyr1068) and Total EGFR ELISA Kit (RayBiotech).

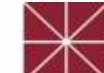

CHAPMAN

UNIVERSITY

SCHOOL OF PHARMACY

\section{RESULTS}

$\triangle S W O G$

$>$ Highest vitamin K1 level $=8.22 \mathrm{ng} / \mathrm{ml}$ in plasma at $50 \mathrm{hrs}$ in healthy skin model.

$>$ Overall, mean plasma vitamin $\mathrm{K} 1$ levels were higher than mean serum vitamin $\mathrm{K} 1$ levels (1.87 [1.43, 2.32] vs. $1.10[0.66$, 1.55], $p=0.017)$.

$>$ MANOVA did not show significant differences in mean vitamin $\mathrm{K} 1$ levels over time for serum or plasma $(\mathrm{p}=0.175)$ in healthy or inflamed skin $(p=0.966)$.

$>$ Overall, healthy skin vitamin K1 levels were not significantly different than inflamed skin vitamin $\mathrm{K} 1$ levels (1.47 [1.03, 1.92] vs. $1.50[1.03$, 1.97], $p=0.930$ ) .
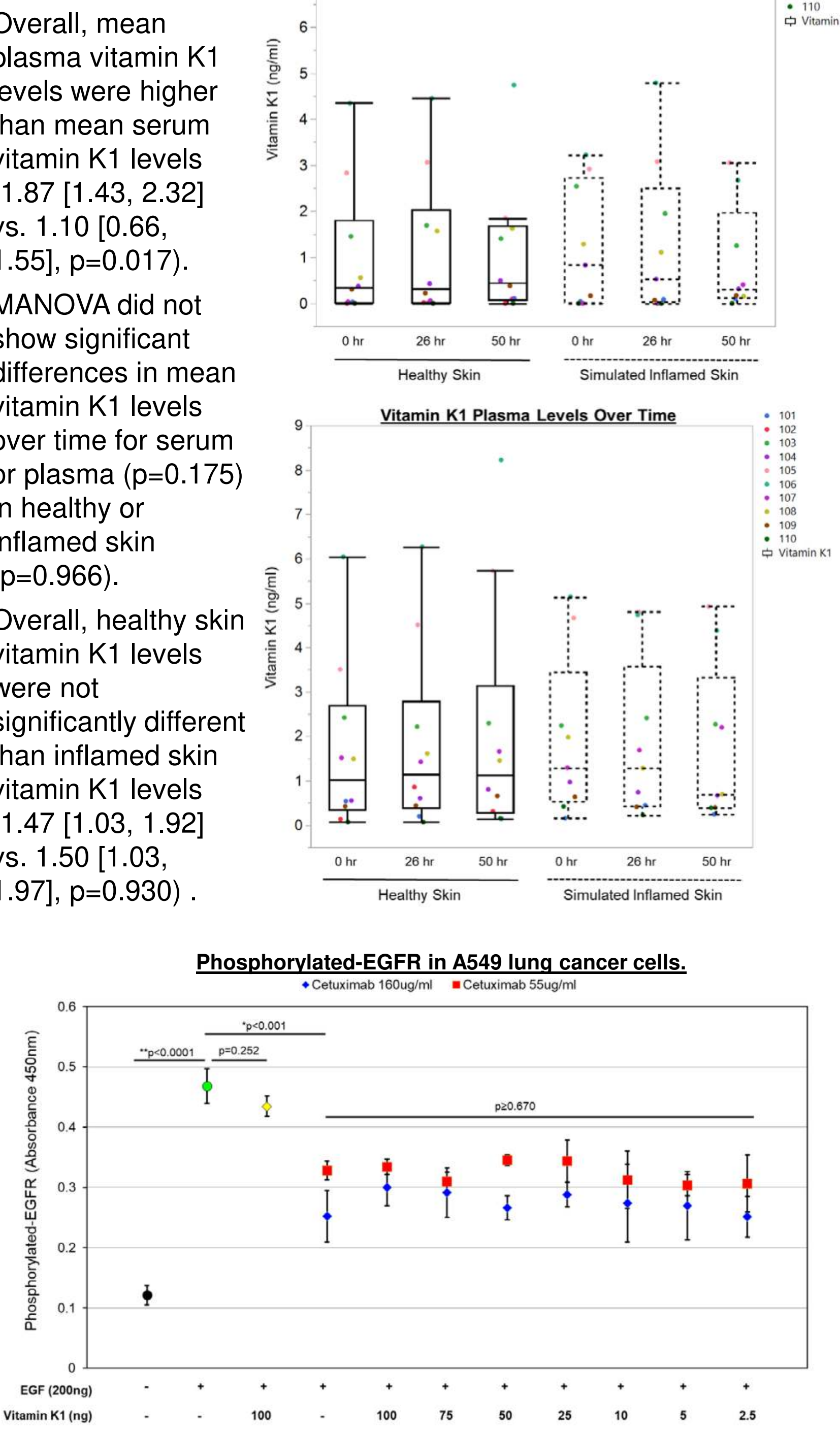

\section{CONCLUSIONS}

$>$ Application of topical $0.1 \%$ vitamin $\mathrm{K} 1$ product to healthy or simulated inflamed skin did not result in significant increases in vitamin $\mathrm{K} 1$ levels in serum or plasma.

$>$ Vitamin $\mathrm{K} 1$ (2.5ng -100ng) did not interfere with cetuximabinhibition of EGFR in A549 lung cancer cells.

$>$ Our topical $0.1 \%$ vitamin $\mathrm{K} 1$ product appears safe for use in patient receiving EGFRI therapy. 